PHYSICAL REVIEW D 91, 119905(E) (2015)

\title{
Erratum: Limiting Lorentz violation from neutron-antineutron oscillation [Phys. Rev. D 91, 096009 (2015)]
}

K. S. Babu and Rabindra N. Mohapatra

(Received 1 June 2015; published 9 June 2015)

DOI: 10.1103/PhysRevD.91.119905

PACS numbers: $11.30 . \mathrm{Cp}, 99.10 . \mathrm{Cd}$

Following are corrections to the article.

(1) The second line after Eq. (1) should read $\tau_{n-\bar{n}} \geq 0.8 \times 10^{8}$ sec and $\delta_{B=2} \leq 10^{-33} \mathrm{GeV}$.

(2) On page 2, second column, third line from bottom, $P_{n-\bar{n}}<10^{-8}$ should read $P_{n-\bar{n}}<10^{-18}$.

(3) On page 3, second column, fifth line from the end of the section, $10^{-28}$ should read $10^{-33}$.

(4) It should be noted that whenever LIV operators violate rotational invariance (e.g. lines 2, 5, 7, and 8 in Table I), they are different from the ones that involve the time component only and should be observable by other means in the laboratory.

The final conclusions of the paper remain unchanged.

\section{ACKNOWLEDGMENTS}

We thank Yuri Kamyshkov for pointing out the numerical typos and Arkady Vainshtein for the comment (4). 